\title{
Medicinal plants of Farashband tribe's winter pastures and their traditional uses
}

\author{
Seyed Hassan Zali ${ }^{1 *}$, Rahbar Tahmasb ${ }^{2}$ \\ ${ }^{1,2}$ Sari Agricultural Sciences \& Natural Resources University, Moji, Iran
}

Key Words:

Medicinal plants

Farashband tribe

Traditional uses

Asteraceae

Chenopodiaceae

Received: 15 February 2015

Accepted: 26 July 2015

Published: 22 February 2016

\begin{abstract}
Medicinal plants are a large group of plants used to prevent and or treat human and animal diseases. The World Health Organization (WHO) estimates that 80 percent of some Asian and African countries presently use herbal medicine for some aspect of primary health care. Since migrating nomads have more connection with nature and on many days of the year they are far from the cities, therefore they take medicinal plants more than other people. By several trips to study areas, 134 plant species distributed in 97 genera and 37 families were collected and identified. Based on the exhaustive interviews with indigenous people and medicinal plants, these plants' medicinal plants were listed. Investigations have resulted that 67 species of these plants have medicinal uses. These medicinal species belong to 31 families. Asteraceae with 11 species, Chenopodiaceae with seven species, and Lamiaceae with six species are the most important medicinal plant families. The major life forms of the medicinal plants in this area were annual herbs followed by perennial trees and shrubs with a proportion of $34.32 \%$ and $26.86 \%$, respectively. $17.91 \%$ of the species are perennial herbs, $14.92 \%$ are perennial bushes, and $5.97 \%$ are annual bushes. Our results showed leaf and flower have the most traditional uses on the Farashband tribe, with a proportion of $62.69 \%$ and $34.33 \%$.
\end{abstract}

(C) 2016 The Author(s). Published by TAF Publishing.

\section{INTRODUCTION}

Plants have been the basis for medical treatments through much of human history, and such traditional medicine is still widely practiced today. Although currently, most of the new medicines are chemicals, approximately $30 \%$ of medicinal products have plant origin [1]. The WHO estimates that 80 percent of the populations of some Asian and African countries presently use herbal medicine for some aspect of primary health care [2]. Pharmaceuticals are prohibitively expensive for most of the world's population, half of which lived on less than $\$ 2$ U.S. per day in 2002 [3]. In comparison, herbal medicines can be grown from seed or gathered from nature for little or no cost. Native Americans medicinally used about 2,500 of the approximately 20,000 plant species that are native to North America [4]. Tribal and folk medicinal practices have medicinal herbs and other forest products as their base and comprise the largest part of primary health care in south Asia even today.

Improvement of technology, civilization and usage of unnatural fast-food caused increase gap of new generation and nature. Hence, uses of knowledge and experience native people that day by day being forgotten is necessary [5]. Looking at the four thou-

\footnotetext{
${ }^{*}$ Corresponding author: Seyed Hassan Zali

${ }^{\dagger}$ Email: hassan.zali@gmail.com
} 
sand years' written history of plants used to treat diseases shows that it has many ups and downs and over the centuries it has evolved. At first, some healers only knew that some leaves can soften the wounds or heal it faster but over the time; they found more information in this case. In fact, knowledge of plants uses is as old as human civilization and culture and almost all groups and tribes benefited from plants which are grown around them [6]. With the advancement of science and new discoveries in biochemistry and physiology of plants components, beauty and health care as well as medical science has gone step by step to the herbalism and medical plant uses. Yineger et al. [7] studied traditional medicinal plant knowledge and use by local healers in Sekoru District, Jimma Zone, Southwestern Ethiopia, they were reported that 27 plant species belonging to 27 genera and 18 families were commonly used to treat various human ailments. They found most of studied species (85.71\%) were wild and harvested mainly for their leaves (64.52\%). The most cited ethno medicinal plant species was Alysicarpus quartinianus [7]. Teklehaymanot et al. [9] in a study entitled Ethno botanical study of medicinal plants used by people in Zegie Peninsula, Northwestern Ethiopia reported that sixty-seven medicinal plants used as a cure for 52 ailments. They are distributed across 42 families and 64 genera. They described that the most frequently utilized plant part was the underground part (root/rhizome/bulb) (42\%) and the largest number of remedies was used to treat gastrointestinal disorder and parasites infections (22.8\%) followed by external injuries and parasites infections (22.1\%). They found the administration routes are oral (51.4\%), external (38.6\%), nasal (7.9\%), and ear (2.1\%) [9]. Sartavi et al. [10] in Bushehr province of Iran collected 70 species of medicinal plants.

They reported: the most important medicinal plants families in the province are: Cruciferae, Umbelliferae, Compositae, and Labiatae [10]. Alavi [11] in a study on the folk uses of wild plants in northern Iran found that 35 species of wild plants used by the people as medicinal plants. In an ethnobotanical study of medicinal plants marketed in La Paz and El Alto cities in the Bolivian Andes, Macía et al. [12] reported medicinal information for about 129 species, belonging to 55 vascular plant families and one uncertain lichen family. The most important family was Asteraceae with 22 species, followed by Fabaceae with 11, and Solanaceae with eight. They found most remedies were prepared from a single species, however some applications were always prepared with a mixture of plants, e.g. for abortion, and the complex of luxation and swellings. The part of the plant most frequently used was the aerial part (29.3\%) and the leaves $(20.7 \%)$. The remedies were mainly prepared as a decoction (47.5\%) and an infusion (28.6\%) [12].

\section{MAT ERIAL AND METHOD}

\section{Study Region}

Farashband city is located at $172 \mathrm{~km}$ west of Shiraz in Fars province in Iran. The city is limited from north to Koohmareh-Sorkhy, from East to Firooz-abad, from South to Lar and from west to Ahrom of Bushehr province. It is located in a longitude of $51^{\circ}, 30^{\prime}$ to $52^{\circ}, 31^{\prime}$ east and a latitude $28^{\circ}, 00^{\prime}$ to $29^{\circ}, 28^{\prime}$ north, with a surface area of 59455 Hectare. The climate of the study area is hot-arid desert climate. Its average annual temperature of is over 25 and annual precipitation is about 250 to $300 \mathrm{~mm}$. August with an average temperature of $36.7^{\circ} \mathrm{C}$ is the hottest month and January and February with an average temperature of $13.5^{\circ} \mathrm{C}$ and $13.7^{\circ} \mathrm{C}$ are the coldest months of the year. Average of maximum rainfall in the period 27 years occurred in January with $70.1 \mathrm{~mm}$ and average of minimum rainfall with zero mm belong to July and August. Kheir-abad, Mongarak, Chah-gezi, Roo-hoony, Bermeh, Khormayek, Pahna-pahn and Balout-abad located on around of Farashband city 
are the Farashband tribe's winter pastures.

\section{Method}

To identify the plants species of the study are all species were collected from the area, then transferred to the plant laboratory of Sari agricultural sciences and Natural Resources University and with the flora references and experiences of author were identified accurately.

Sake to introduce medicinal plants species of the study area questionnaire and interviews data were collected from Farashb and tribe. Then common name of plants that people were wrote in the questionnaire form or told in interviews as medicinal plants identified using varying medicinal plants and traditional medicine references. Some of unknown plants that by this method did not identify by help of some experts was collected and compared with the collected plants in the plant laboratory. Also, the sites of species distribution, their medicinal properties, and their useable segments were surveyed using various sources. Ninety seven people-aged between 40 to 70 years-individually and or in groups were interviewed and the questionnaire forms were completed with them or interviewer. Interviews continued until the repetitive response was demonstrated for the researcher and the interviewer will not add anything new.

\section{RESULT S}

By several trips to study areas a total of 134 plant species distributed in 97 genera and 37 families were collected and identified. Summing up questionnaire forms and interviews were resulted that 67 species of these plants have medicinal uses. These medicinal species belongs to 58 genera of 31 families. Among the organs, leaves that use as medicine in 47 species have the most consumption. In 32 species flowers, in 21 species stems, in 8 species aerial parts of plant and in 7 species roots have medicinal usages. Gum-that uses in 4 species for medicinal purposes-has the lowest consumption (Figure 1).

These medicinal plants frequently use for treat to stomach disease, colds, digestive disorders, psychiatric disorders, vomiting. The most usage method of these medicinal plants among the native tribes of the study area is decoction and brew. The most frequently of medicinal plants were seen in 3 families Asteraceae (with 11 species), Chenopodiaceae (with 7 species) and Lamiaceae (with 6 species). 27 species of the medicinal plants are annual herbs, 18 species are trees and shrubs, 12 species are perennial herbs and 10 species are forbs (Figure 2).

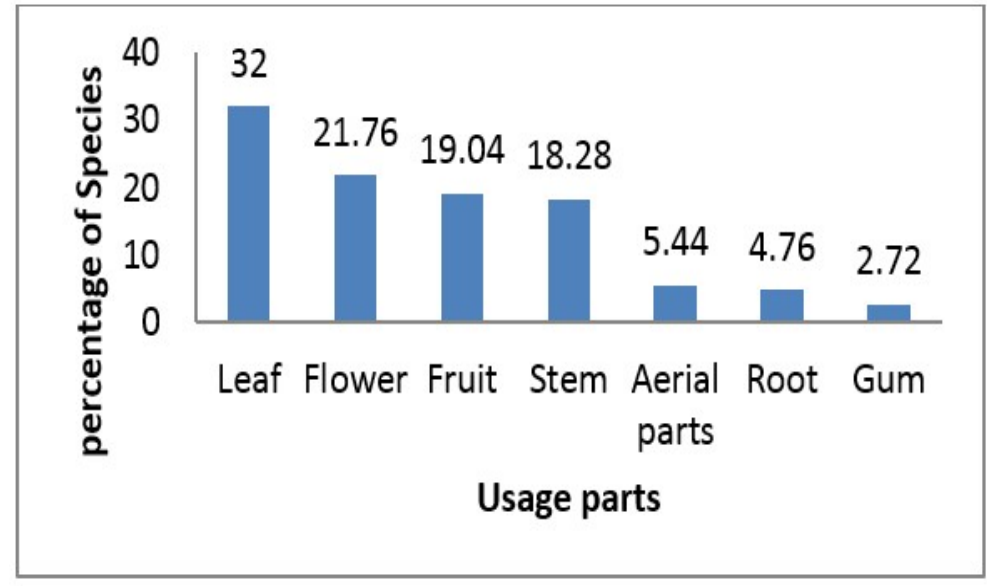

FIGURE 1. Species frequency with respect to their useable organs 


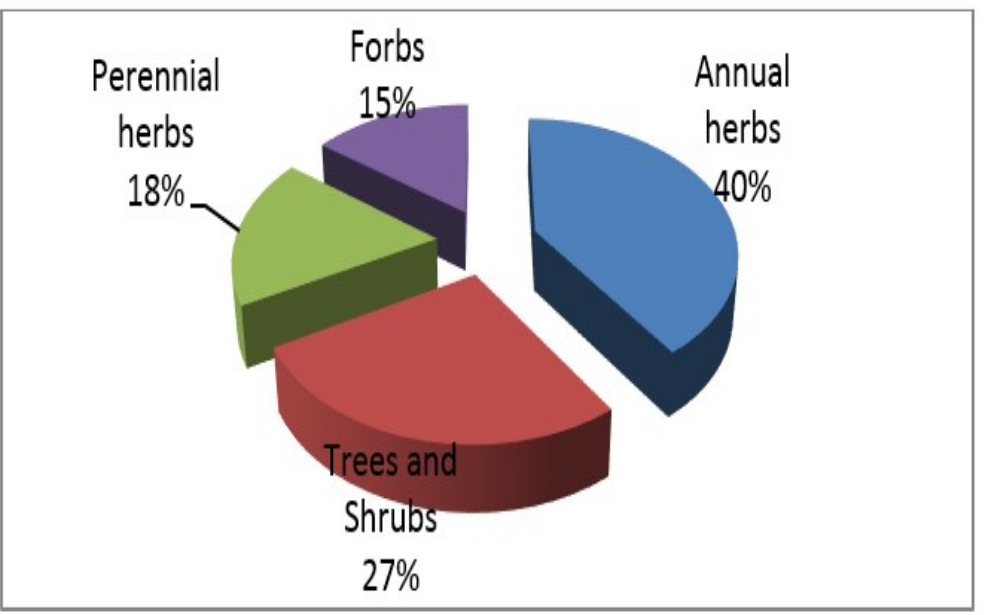

FIGURE 2 . Percentage of the species based on their vegetation period

TABLE 1. Botanical properties of medicinal plants of Farashband tribe's winter pastures, their usable parts \& traditional uses

\begin{tabular}{|c|c|c|c|c|c|c|c|c|}
\hline & Species & Family & Local Name & Persian Name & $\begin{array}{l}\text { Useable } \\
\text { Parts* }\end{array}$ & $\begin{array}{l}\text { Vege- } \\
\text { tative } \\
\text { Form** }\end{array}$ & Traditional Uses & Method of Use \\
\hline 1 & Acer cinerascens & Acaraceae & Keikoom & Keikom & $\mathrm{Fr}$ & $\mathrm{T}$ & $\begin{array}{l}\text { Memory Booster, Distrac- } \\
\text { tion therapy, Intelligence, } \\
\text { Performance-enhancing }\end{array}$ & Orally \\
\hline 2 & 'óóo Achilla Millefilium & Astraceae & Sari Gool, Berenjas & Boomadarn mahalli & $\mathrm{L}, \mathrm{F}, \mathrm{S}$ & $\mathrm{F}$ & $\begin{array}{l}\text { Treat to: Diarrhea, Ner- } \\
\text { vous system diseases, Dia- } \\
\text { betes, Hypertension, Blood } \\
\text { fat }\end{array}$ & Decoction \& Brew \\
\hline 3 & Achilla santolina & Astraceae & Berenjas & Boomadarn & $\mathrm{L}, \mathrm{F}, \mathrm{S}$ & $\mathrm{AH}$ & $\begin{array}{l}\text { Bellyache, Diarrhea, Infec- } \\
\text { tious, Anti-stimulation of } \\
\text { stomach, Diabetes }\end{array}$ & Decoction \& Brew \\
\hline 4 & Alcea aucheri & Malvaceae & - & Gol Khatmi & $\mathrm{L}, \mathrm{F}$ & $\mathrm{PH}$ & $\begin{array}{l}\text { Colds, Influenza, Sore } \\
\text { throat }\end{array}$ & Decoction \\
\hline 5 & Alyssum Spp & Crucifereae & - & Ghoddomeh Shirazi & Fr,L & $\mathrm{AH}$ & Stomachic, Fattening drug & Brew \\
\hline 6 & Amygdalus haussknechtii & Rosaceae & Arjeen & Arjanak & $\mathrm{L}, \mathrm{F}$ & S & Anti-Hair Loss, Hair color & External with Henna \\
\hline 7 & Amygdalus Lycioides & Rosaceae & Chali & Tangers & $\mathrm{L}, \mathrm{F}, \mathrm{Fr}$ & S & $\begin{array}{l}\text { Anti-Hair Loss, Relieve } \\
\text { hoarseness }\end{array}$ & External with Henna \\
\hline 8 & Amygdalus Scoparia & Rosaceae & Berogh & Badam Koohi & $\mathrm{Fr}, \mathrm{G}$ & $\mathrm{T}$ & $\begin{array}{l}\text { Toothache, Relieve hoarse- } \\
\text { ness }\end{array}$ & $\mathrm{Fr}=$ Decoction, $\mathrm{G}=$ Placed on the tooth \\
\hline 9 & Anabasis Aphylla & Chenopodiacea & Oldorok & Shepesho & A & PH & Insect repellent & Hung from the ceiling \\
\hline 10 & Anthemis nobilis & Astraceae & Momonak & Babooneh & $\mathrm{L}, \mathrm{F}, \mathrm{S}$ & $\mathrm{AH}$ & $\begin{array}{l}\text { Psychiatry, Colds, An- } \\
\text { tidepressants Anorexia } \\
\text { Treatment Anti-Fever, } \\
\text { Anti-asthma, Tonic }\end{array}$ & Decoction \& Brew \\
\hline 11 & Artemisia aucheri & Astraceae & Youshagh & Dermaneh Koohi & $\mathrm{L}, \mathrm{F}, \mathrm{S}$ & $\mathrm{PH}$ & Anti-parasitic, Digestive & Decoction \& Brew \\
\hline 12 & Artemisia sieberi & Astraceae & Youshagh & Dermaneh Dashti & $\mathrm{L}, \mathrm{F}, \mathrm{S}$ & $\mathrm{F}$ & $\begin{array}{l}\text { Anti-parasitic, } \\
\text { Helminthic }\end{array}$ & Decoction \& Brew \\
\hline 13 & Calotropis perocera & Asclepiadacea & Estabragh & Estabragh & $\mathrm{R}$ & S & Anti-Fever & Decoction \\
\hline 14 & Capparis Capparis var.muronifolia & Capparidacea & Lageji & Kavar & $\mathrm{Fr}, \mathrm{B}$ & $\mathrm{F}$ & $\begin{array}{l}\text { Breast-pain, Kidney infec- } \\
\text { tion, Anti-anemia }\end{array}$ & Decoction, Orally \\
\hline 15 & Capparis Spinosa & Capparidacea & Lageji & Kavar & $\mathrm{Fr}$ & $\mathrm{F}$ & Dizziness, Anti-anemia & Orally \\
\hline 16 & Cardaria Draba & Crucifereae & Moocheh & Ozmak & A & $\mathrm{AH}$ & $\begin{array}{l}\text { Astringent, Prevent of } \\
\text { stomach and uterus bleed- } \\
\text { ing, Kidney disease }\end{array}$ & Decoction \\
\hline 17 & Chenopodium Album & Chenopodiacea & Salimeh & Salmeh Tareh & $\mathrm{L}, \mathrm{F}, \mathrm{S}$ & $\mathrm{AH}$ & $\begin{array}{l}\text { Antinausea, Bellyache, } \\
\text { Stomach cramps }\end{array}$ & Orally \\
\hline 18 & Chrozophora tinctoria & Euphorbiaceae & Chabla & Goosh bareh & $\mathrm{L}, \mathrm{F}$ & $\mathrm{AH}$ & Earache, Sore throat, Colds & Incense \\
\hline 19 & Citrullus schrad & Cucurbitacea & Achi Gharpose & Hendevaneh abujahle & $\mathrm{Fr}, \mathrm{Se}$ & $\mathrm{AH}$ & $\begin{array}{l}\text { Hypertension, Anti- } \\
\text { parasitic, Hyperlipidemia, } \\
\text { Breast-pain Diabetes }\end{array}$ & Orally, Rub \\
\hline 20 & Cotoneaster Persica & Rosaceae & Mordar Aghaji & Shir khesht & $\mathrm{L}, \mathrm{Fr}$ & S & Baby Jaundice, Anti-Fever & Wash, Incense \\
\hline 21 & Cynodon dactylon & Poaceae & Margh & Margh & A & $\mathrm{PH}$ & $\begin{array}{l}\text { Digestive, Antitussive, Di- } \\
\text { arrhea, Stomach cramps }\end{array}$ & $\mathrm{Fr}=$ Orally, Other $=$ Decoction \\
\hline 22 & Dianthus tabrizianus & Caryophyllaceae & Mikhak & Mikhak & $\mathrm{Fr}$ & S & $\begin{array}{l}\text { Toothache, Body Perfume, } \\
\text { Mouth odor removal, Eye- } \\
\text { sight Improvement }\end{array}$ & Placed on the tooth \\
\hline 23 & Echinops Aucheri & Astraceae & Shekar tighool & Shekar tighal & $\mathrm{L}, \mathrm{S}$ & $\mathrm{F}$ & $\begin{array}{l}\text { Relieve hoarseness, Sore } \\
\text { throat, Antitussive }\end{array}$ & $\mathrm{Fr}=$ Orally, Other $=$ Decoction \\
\hline
\end{tabular}


TABLE 1. continue.....

\begin{tabular}{|c|c|c|c|c|c|c|c|c|}
\hline 24 & Ephedra foliata & Ephedraceae & Pazan soghli & Rish boz & $\mathrm{L}$ & $\mathrm{PH}$ & Bracing, Anti-Fever, & Orally, Brew \\
\hline 25 & Ephedra procera & Ephedraceae & Pazan soghli & Ephedra & A & $\mathrm{PH}$ & Anti-asthma & Decoction \\
\hline 26 & Eruca Sativa & Brassicaceae & Khardel & Mandab & $\mathrm{Se}$ & $\mathrm{AH}$ & Sexuality enhancement & Orally \\
\hline 27 & Eryngium billardieri & Apiacea & Zol & Zool & S & $\mathrm{PH}$ & Skin Moisturizer & Smoke \\
\hline 28 & Ficus Carica & Moraceae & Anjeal & Anjeer & $\mathrm{Fr}$ & $\mathrm{T}$ & $\begin{array}{l}\text { Memory Improvement, } \\
\text { Anti-anemia Intelligence, } \\
\text { Increaser }\end{array}$ & Orally \\
\hline 29 & Fraxinus rotundifolia & Oleacea & Ghoosh euzimi & Zaban Gonjeshk & $\mathrm{Fr}$ & $\mathrm{PH}$ & Anti-Caries & Placed on the tooth \\
\hline 30 & Frulago angulata & Umbelliferae & Chool & Chovile & $\mathrm{L}, \mathrm{F}, \mathrm{S}$ & $\mathrm{AH}$ & $\begin{array}{l}\text { Digestive diseases, Home } \\
\text { Perfume, Anti-stimulation } \\
\text { of stomach }\end{array}$ & Orally \\
\hline 31 & Fumaria asepala & Fumariaceae & Shatareh & Shah Tareh & A & $\mathrm{AH}$ & $\begin{array}{l}\text { Treat of Itching, Hyperlipi- } \\
\text { demia, Jaundice, }\end{array}$ & Decoction \\
\hline 32 & Glycyrrhiza glabra & Fabaceae & Pian(Mag) & Shirin bayan & $\mathrm{R}$ & $\mathrm{F}$ & $\begin{array}{lr}\text { Colds, Sore } & \text { throat, An- } \\
\text { titussive, } & \text { Psychiatry } \\
\text { Anti-asthma, } & \text { Banged } \\
\text { remediation } & \end{array}$ & $\mathrm{R}=$ Decoction, Orally \\
\hline 33 & Gundelia tournefortii & Astraceae & Kangar & Kangar & $\mathrm{L}, \mathrm{R}$ & $\mathrm{F}$ & $\begin{array}{lr}\text { Stomach } & \text { booster, } \\
\text { Heartache, Jaundice, } \\
\text { Digestive, Colds }\end{array}$ & $\mathrm{R}=$ Orally, $\mathrm{L}=\mathrm{Brew}$ \\
\hline 34 & Halocnemum strobilaceum & Chenopodiacea & Shoora & Alaf shoor & A & $\mathrm{F}$ & $\begin{array}{l}\text { Digestive, Anti-Headache, } \\
\text { Expectorants }\end{array}$ & Decoction \& Brew \\
\hline 35 & Hypericum Perforatum & Clusiaceae & Hoolileh & Chayeh koohi & $\mathrm{L}, \mathrm{F}, \mathrm{S}$ & $\mathrm{AH}$ & Dizziness, Psychiatry & Decoction \& Brew \\
\hline 36 & Lactuca ativa & Astraceae & Aghja ghoyogh & Kahoyeh vahshi & $\mathrm{L}, \mathrm{G}$ & $\mathrm{F}$ & $\begin{array}{l}\text { Stomachic, Sedative, Ear- } \\
\text { ache, }\end{array}$ & $\mathrm{L}=$ Orally, G=Rub \\
\hline 37 & Malva parvilflora & Malvaceae & Toola & Panirak & $\mathrm{L}, \mathrm{Fr}$ & $\mathrm{AH}$ & $\begin{array}{l}\text { Kidney pain, Infectious, } \\
\text { Colds, Earache, Anti- } \\
\text { inflammation, Relieve } \\
\text { hoarseness, Digestive }\end{array}$ & Brew, Incense \\
\hline 38 & Matricaria chamomilla & Astraceae & Moomoonak & Babooneh & $\mathrm{L}, \mathrm{F}, \mathrm{S}$ & $\mathrm{AH}$ & $\begin{array}{l}\text { Anti-parasitic, Hyper- } \\
\text { lipidemia, Hypertension, } \\
\text { Diabetes, Menstrual stim- } \\
\text { ulant, Tonic, Psychiatry }\end{array}$ & Decoction \& Brew \\
\hline 39 & Menta longifolia & Lamiaceae & Yarpose & Pooneh & $\mathrm{L}, \mathrm{F}$ & PH & $\begin{array}{l}\text { Antitussive, Anti- } \\
\text { heartburn, Bellyache, } \\
\text { Anti-Vomiting, Sore throat, } \\
\text { Carminative }\end{array}$ & o Orally, Decoction \\
\hline 40 & Mentha spicata & Lamiaceae & Dagh Yarposi & Pooneh kohi & $\mathrm{L}, \mathrm{F}$ & $\mathrm{AH}$ & $\begin{array}{l}\text { Diarrhea, Mouth odor } \\
\text { removal, Anti-Vomiting, } \\
\text { Bellyache }\end{array}$ & Orally, Decoction, Hung from the ceiling \\
\hline 41 & Myrtus communis & Myrtaceae & Moord & Moord & $\mathrm{S}, \mathrm{L}$ & $\mathrm{S}$ & Foot & Body \& Mouth odor removal \& Gargle, Wash \\
\hline 42 & Nerium indicu & Apocynaceae & Khar zahleh & Khar zahreh & $\mathrm{L}$ & S & Anti-Dandruff, Heartache & Decoction \\
\hline 43 & Oliveria decumbens & Umbelliferae & - & Dan danak & A & $\mathrm{AH}$ & $\begin{array}{l}\text { Stomachache, Digestive, } \\
\text { Carminative }\end{array}$ & Decoction \& Brew \\
\hline 44 & Papaver tenuifolium & Papaveraceae & Norooz gooli & Shaghayegh vahshi & L & $\mathrm{AH}$ & Bracing, Psychiatry & Brew \\
\hline 45 & peganum harmala & Zygophyllaceae & Euzerlik & Esfand & $\mathrm{L}, \mathrm{F}, \mathrm{S}, \mathrm{Fr}$ & $\mathrm{F}$ & Disinfectants & Smoke \\
\hline 46 & Perosopis Fracta & Papilionaceae & - & Kahoorak & $\mathrm{R}, \mathrm{L}$ & $\mathrm{T}$ & $\begin{array}{l}\text { Diuretics, laxative, Lower- } \\
\text { ing blood }\end{array}$ & viscosity Brew \\
\hline 47 & phlomis aucheri & Lamiaceae & Ghoozi ghoolag & Goosh bareh & $\mathrm{L}, \mathrm{F}$ & PH & $\begin{array}{l}\text { Acne Removal, Anti-Fever, } \\
\text { Colds }\end{array}$ & Incense \\
\hline 48 & Pistacia atlantica & Anacardiaceae & Ban & Pesteh vahshi & $\mathrm{R}, \mathrm{Fr}, \mathrm{G}$ & $\mathrm{T}$ & $\begin{array}{l}\text { Antitussive, Colds, Sore } \\
\text { throat, Relieve hoarseness, } \\
\text { Knee Pain remediation, } \\
\text { Jaundice, Toothache, Anti- } \\
\text { Caries, Treat of feet or } \\
\text { hand Cracking, Expecto- } \\
\text { rants, Digestive }\end{array}$ & Decoction, External, Chew \\
\hline 49 & Pistacia Khynjuk & Anacardiaceae & Koolookhoon & Kal khonak & $\mathrm{L}, \mathrm{Fr}, \mathrm{R}, \mathrm{G}, \mathrm{B}$ & $\mathrm{T}$ & $\begin{array}{l}\text { Antitussive, Colds, Sore } \\
\text { throat, Relieve hoarseness, } \\
\text { Knee Pain remediation, } \\
\text { Menstrual stimulant, } \\
\text { Toothache }\end{array}$ & $\mathrm{L}=$ Incense, $\mathrm{B}=$ Decoction, $\mathrm{Fr}=$ Orally, $\mathrm{G}=$ Poultice \\
\hline 50 & Rumex chalepensis Miller & Polygonaceae & Toroshak & Torshak & $\mathrm{L}, \mathrm{F}, \mathrm{S}$ & $\mathrm{AH}$ & Antinausea, Expectorants & Orally \\
\hline 51 & Rumex deritatus & Polygonaceae & Toroshak & Torshak dandanehdar & $\mathrm{L}, \mathrm{F}, \mathrm{S}$ & $\mathrm{AH}$ & $\begin{array}{l}\text { Antinausea, Vomiting of } \\
\text { Pregnancy period, Colds, } \\
\text { Stomachic, Bracing }\end{array}$ & $\mathrm{L}=$ Brew, Other= Orally \\
\hline 52 & Salsola Crassa & Chenopodiacea & - & Shoor alvan & A & $\mathrm{F}$ & laxative, Bladder disease & Orally, Decoction \\
\hline 53 & Scariola orientalis & Astraceae & - & Gav chagh kon & $\mathrm{L}$ & $\mathrm{AH}$ & Diabetes, Hyperlipidemia & Orally \\
\hline 54 & secal montanum & Poaceae & - & Chavdar & $\mathrm{Fr}$ & PH & $\begin{array}{l}\text { Hypertension, Diabetes, } \\
\text { Heart Strengthen }\end{array}$ & Orally \\
\hline 55 & Seidlitzia rosmarinus & Chenopodiacea & Chaghan & Oshnian & $\mathrm{L}, \mathrm{F}$ & S & $\begin{array}{l}\text { Anti-Dandruff, Anti-Hair } \\
\text { Loss }\end{array}$ & Decoction \\
\hline 56 & Spinacia oleracea & Chenopdiaceae & Esfenaj & Esfenaj & $\mathrm{L}, \mathrm{S}$ & $\mathrm{AH}$ & Stomach booster, Digestive & Orally \\
\hline 57 & Suaeda aegiptica & Chenopodiacea & - & Siah shooreh mesri & $\mathrm{L}$ & $\mathrm{F}$ & Sedative, Expectorants & Brew \\
\hline 58 & Tanacetum parthenium & Astraceae & Sighirmomonaki & Babooneh gavi & $\mathrm{L}, \mathrm{F}, \mathrm{S}$ & $\mathrm{AH}$ & $\begin{array}{l}\text { Psychiatry, Sedative, } \\
\text { Digestive }\end{array}$ & Decoction \& Brew \\
\hline
\end{tabular}


TABLE 1. continue....

\begin{tabular}{|c|c|c|c|c|c|c|c|c|}
\hline 59 & Teucrium Polium & Lamiaceae & Halpeh & Kalpooreh & $\mathrm{L}, \mathrm{F}$ & $\mathrm{F}$ & $\begin{array}{l}\text { Stomachic, Colds, Hyper- } \\
\text { tension, Heartache Anti- } \\
\text { Headache, Anti-Fever }\end{array}$ & Decoction \& Powder \\
\hline 60 & Thymus Vugaris & Lamiaceae & Oshom & Avishan & $\mathrm{L}, \mathrm{F}, \mathrm{S}$ & $\mathrm{F}$ & $\begin{array}{l}\text { Digestive, Colds, Psychi- } \\
\text { atry Anti-Vomiting, Anti- } \\
\text { poisoning }\end{array}$ & Decoction, Brew, Orally \\
\hline 61 & Tribulus trrestris & Zygophylaceae & - & Khar khasak & $\mathrm{L}, \mathrm{Fr}$ & $\mathrm{AH}$ & $\begin{array}{l}\text { Hypertension, Hyperlipi- } \\
\text { demia, Diabetes }\end{array}$ & Fr=Orally, $\mathrm{L}=$ Brew \\
\hline 62 & Urtica Dioica & Urticaceae & Gazgazak & Gazaneh & $\mathrm{L}, \mathrm{S}$ & $\mathrm{AH}$ & Foot \& Body odor removal & Fresh or Decoction into the shoes \\
\hline 63 & Vitex psedo-negundo & Verbenaceae & Beniroo & Bengro & Fr & $\mathrm{S}$ & Anti-heartburn & Orally \\
\hline 64 & Zataria multiflora & Lamiaceae & Oshom & Avishan Shirazi & $\mathrm{L}, \mathrm{F}, \mathrm{S}$ & $\mathrm{F}$ & $\begin{array}{l}\text { Distraction therapy, Intel- } \\
\text { ligence Increaser, Sedative, } \\
\text { Menstrual stimulant, } \\
\text { Stomachic, Carminative }\end{array}$ & Decoction \& Powder \\
\hline 65 & Ziziphus nummularia & Rhamnaceae & Rimlik & Remlik & $\mathrm{L}, \mathrm{Fr}, \mathrm{R}$ & $\mathrm{S}$ & $\begin{array}{l}\text { Digestive, Anti-heartburn, } \\
\text { Antinausea, Anti-gastritis, } \\
\text { Anti-Hair Loss, Anti- } \\
\text { Dandruff }\end{array}$ & $\mathrm{R}=$ Decoction, Other $=$ Orally \\
\hline 66 & Ziziphus Spinachriti i & Rhamnaceae & Koonar & Konar & $\mathrm{L}, \mathrm{Fr}, \mathrm{R}$ & $\mathrm{S}$ & $\begin{array}{l}\text { Digestive, Anti-heartburn, } \\
\text { Antinausea, Anti-gastritis, } \\
\text { Hair-Strengthen, Anti- } \\
\text { Dandruff, Anti-Hair Loss }\end{array}$ & $\mathrm{R}=$ Decoction, $\mathrm{L} \& \mathrm{Fr}=$ with Henna, Orally \\
\hline 67 & Zygophyllum propinguum & Zygophyllaceae & - & Gheech & $\mathrm{S}$ & $\mathrm{S}$ & Anti-Stomach worm & Decoction \\
\hline
\end{tabular}

* Fr=Fruit, L=Leaf, F=Flower, R= Root, S=Stem, G=Gum, B=Bark, A=Aerial parts, Se=Seed

** $\mathrm{S}=\mathrm{Shrub}, \mathrm{T}=$ Tree, $\mathrm{AH}=$ Annual Herb, $\mathrm{F}=$ Forb

\section{REFERENCES}

1. Yuan R, Lin, Y. Traditional Chinese medicine: An approach to scientific proof and clinical validation. Pharmacology \& Therapeutics. 2000; 86(2): 191-198. DOI: 10.1016/S0163-7258(00)00039-5

2. Holtz C. Global health care: Issues and policies. 2nd ed. New York, NY: Jones \& Bartlett Publishers; 2012.

3. DaSilva EJ, Baydoun E, Badran A. Biotechnology and the developing world. Electronic Journal of Biotechnology. 2002; 5(1): 64-92. DOI: $10.2225 /$ vol5-issue1-fulltext-1

4. Selin, H. ed. Encyclopaedia of the history of science, technology, and medicine in non-Westen cultures. Berlin, DE: Springer Science \& Business Media; 2013.

5. Zali SH. Introducing on the most important medicinal plants of Tyar sub basin with emphasis on composition and therapeutic effects. Paper presented at: 2nd National Conference of Reza's Medicine, Mashhad Medical Sciences University, Mashhad, IR; 2010.

6. Rates SMK. Plants as a source of drugs. Toxicon. 2001; 39(5): 603-613. DOI: 10.1016/S0041-0101(00)00154-9

7. Yineger H, Yewhalaw D. Traditional medicinal plant knowledge and use by local healers in Sekoru District, Jimma Zone, Southwestern Ethiopia. Journal of Ethnobiology \& Ethnomedicine. 2007; 3(24): 1-7. DoI: 10.1186/1746-4269-3-24

8. Giday M, Asfaw Z, Woldu Z. Medicinal, plants of the mein it ethnic grup of Ethiopia. Journal of Ethnophama. 2009; 124(3): 513-521. DOI: $10.1016 /$ j.jep.2009.05.009

9. Teklehaymanot T, Giday M. Ethno botanical study of medicinal plants used by people in Zegie Peninsula, Northwestern Ethiopia. Journal of Ethnobiology \& Ethnomedicine. 2007; 14(3): 1-11.

10. Sartavi K, Gholamian F. Medicinal plants of Boushehr province. Journal of Medicinal and Aromatic Plants Research. 2004; 20(2): 213-227.

11. Alavi SZ. Traditional use of medicinal plants native to North of Iran. Journal of Herbal Medicines. 2011; 2: 113-120.

12. Macía MJ, García E, Vidaurre PJ. An ethnobotanical survey of medicinal plants commercialized in the markets of La Paz and El Alto. Bolivia Journal of Ethnopharmacology. 2005; 97(2): 337-350. DOI: 10.1016/j.jep.2004.11.022 